\title{
Dynamic Binding for BPEL Processes - A Lightweight Approach to Integrate Semantics into Web Services
}

\author{
Ulrich Küster and Birgitta König-Ries \\ Institute of Computer Science, Friedrich-Schiller-Universität Jena \\ D-07743 Jena, Germany \\ ukuester, koenig@informatik.uni-jena.de
}

\begin{abstract}
The area of service oriented computing stretches between two extremes: On the one hand industry has pushed a whole stack of WS-* standards and tools to support the integration of distributed services into business applications. These standards are used in production environments and are applied successfully, e.g. in the area of enterprise application integration. However, the expensive and labor intensive task of putting together services and maintaining and administering the composed applications has to be done manually. In contrast, academia is busily working on numerous efforts leveraging ontology based semantics and various AI planning techniques to automate these tasks. Yet, up to now the developed technologies have rarely if ever been applied in industry. In our opinion, this has two main reasons: there is high cost involved in creating the necessary comprehensive ontologies and businesses are reluctant to trust semantic technologies. In this paper we bring together the extremes in order to combine their strengths. We show how to flexibly integrate advanced semantic service discovery, composition and invocation technology into manually created standard BPEL processes. Our approach leaves it to the discretion of the developer to flexibly choose an appropriate degree of automation for the process at hand and thus offers him complete control over the usage of semantic technology.
\end{abstract}

\section{Introduction}

In recent years, service orientation has evolved as a new paradigm for distributed computing. A whole stack of Web service standards and technologies has been created [12] that forms the maybe most promising implementation of this paradigm. These standards are widely used in production environments and are applied successfully, e.g. in the area of enterprise application integration. Even though web service technology has thus proven to be an effective way of creating widely distributed and loosely coupled systems, the tasks of gluing together the component services and maintaining the composition is still labor intensive and expensive work. Furthermore, the potential of the web service technology cannot be used to full capacity, if service compositions have to be created manually and component services are bound statically in those compositions. To leverage

D. Georgakopoulos et al. (Eds.): ICSOC 2006 Ws, LNCS 4652, pp. 116 127, 2007.

(C) Springer-Verlag Berlin Heidelberg 2007 
the potential availability of thousands of services, automated composition and dynamic discovery and binding are needed.

Academia has thus been working busily on numerous efforts providing ontology based semantic descriptions for services. Based on these descriptions, frameworks are designed to support dynamic service discovery and binding. Various AI planning techniques are applied to (semi-)automatically synthesize service compositions from available component services. Together, these techniques will allow to synthesize an application at runtime from the currently most applicable services in a fully automated fashion. This enables low-effort development of robust, dynamically adaptable applications.

However, up to now, the developed semantic based technologies have rarely if ever - been applied on a large scale in industry. In our opinion, there are two main reasons for this lack of adaptation: First, a prerequisite to using semantic technology is the existence of comprehensive domain ontologies. For most applications, these ontologies do not yet exist. The prospect of having to create them from scratch is rather daunting. The entrance cost for using semantic technology may thus seem forbiddingly high. Second, maybe as a consequence of the AI hype and comparable failure in the past, semantic technologies are regarded with a certain degree of scepticism by many potential users. A widespread -albeit unfounded - lack of trust into the reliability and correctness of these technologies hinders their acceptance.

In this paper, we present an approach to bridge the gap between web service technology used in practice and the promising semantic techniques. This approach enables the gradual integration of semantics-based techniques into existing web service applications. Standard based, well proven web service technology offers stability and control while advanced semantic technology supports dynamic change, adaptability and the potential to take advantage of large numbers of competing service providers. We show how to integrate fully automated service discovery, composition and invocation technology into pre-existing, manually created standard BPEL processes. This leaves it to the discretion of the developer to flexibly choose any appropriate degree of automation within a given process. For instance, a developer may decide to compose modeling intensive tasks or tasks with high security constraints manually, while at the same time using semantic technology for tasks that are highly dynamic or not as expensive to model semantically. We believe that only within such a combination semantic technologies have a chance to overcome the current gap between academia and industry.

Throughout the remainder of the paper, we use the order management system of COS-tec, an imaginary computer online store, as our running example. This order management is currently implemented by a BPEL process. Figure 1 depicts a small cutout of the entire process. Process steps include receiving the order from the customer, checking for item availability and calling a shipment service. In the current BPEL-process, the latter is realized - as is typical for such processes - by a static binding to an external shipment provider. This static binding has a number of disadvantages: If the chosen provider becomes 
temporarily or permanently unavailable, a new provider has to be searched for and the process has to be manually adapted. Also, the chosen provider may not be the cheapest for all types of packages. There might be considerable potential for cost reduction, by choosing the cheapest provider for each individual package. In the current process, this is not done, since the cost for manually comparing prices and manually binding to the appropriate provider in each case may very well outweigh the reduction in shipment cost. Here, semantic technology offers a solution: In our approach, the BPEL process is adapted to calling a semantics-based middleware instead of the shipment provider. This middleware uses a semantic description of the desired shipment to dynamically find and bind the most appropriate shipment provider. This offers both robustness and cost effectiveness. In the remainder of this paper, we will show that using our approach, the effort involved in adapting the BPEL process and thus the cost of leveraging the advantages of semantic technology is very moderate. The rest of

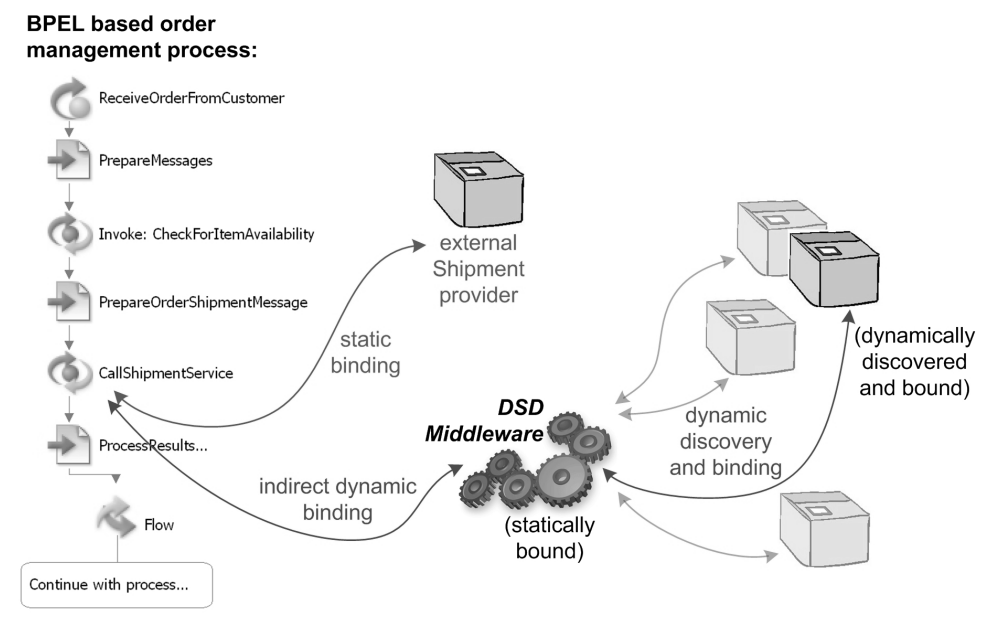

Fig. 1. Dynamic shipment provider discovery and binding for COS-tec's order management BPEL process

this paper is organized as follows: In Section 2 we introduce DSD, the semantic service description language used and the middleware built to support it. In Section 3 we show how to integrate DSD service requests into BPEL processes and how the DSD-Middleware is leveraged to execute these requests when the process runs. In Sections 4 and 5 we evaluate our approach and compare it to the related work and finally in Section 6 we summarize and conclude.

\section{DIANE Service Descriptions}

At the core of all approaches to semantic web services are ontology-based languages that enable the description of service offers and requests and algorithms 
that allow to compare offers and requests in order to find (and bind) the most appropriate service provider for a given offer. The approaches differ in the languages used, the constructs they allow for service descriptions, the information from the descriptions that is taken into account when comparing offers and requests and the degree to which they allow for automatic configuration and binding. This paper uses our DIANE Service Description, DSD [3]. DSD is particularly suitable for the task at hand because of its superior matchmaking capabilities that combine expressiveness with efficient matchmaking and the ability of full automation up to the actual invocation of a discovered service. However, the approach presented could be implemented based on other languages that meet the requirements identified in the evaluation section, too.

In addition to the elements usually found in ontology-based languages, i.e. concepts, their attributes and instances, DSD comprises the following elements geared specifically at capturing the specifics of service descriptions:

- operational elements: Services change the state of the real-world (or the information space). Operational elements allow to express this world-altering capacity. In the example shown in Figure 2, you'll find the operand effect, describing that we are looking for a service that changes the state of the world in such a way, that something is shipped.

- aggregating elements: A service is typically able to offer not one specific effect, but a set of similar effects. A shipment service for instance will be able to offer transport of different kinds of goods from one arbitrary destination to another. That means, services offer to provide one out of a set of effects. Requestors on the other hand, typically are not looking for one specific service, but for any element of the set of services that can provide the desired effect. Sets are depicted in DSD with a small diagonal line in the upper left corner of a concept.

- selecting elements: While a service will offer different effects, the provider will typically allow the requestor to configure the service to choose which of these effects should be provided in a specific instance. In DSD, variables are used to for this task. In our example, the requestor will specify the exact weight, dimension and delivery address upon invocation.

- rating elements: This type of element is used in service requests only. Requestors will typically be willing to invoke services with slightly differing effects. In our example, the requestor is willing to pay any price below 500 USD but would prefer lower prices over higher ones. These preferences can be expressed by rating elements. Rating elements are a key feature of DSD. They allow the requestor to prescribe the desired effect and also acceptable deviations very precisely, thus maximising the likelihood of finding the most appropriate service.

Let us summarize the service request template depicted in Figure 2. COSTec is looking for a service that results in something being shipped from their address to an address that will be specified upon request execution. Weight and dimensions of the good to be shipped will also be filled into the request template as input variables. As an output, COS-Tec expects the price in USD. This price 
should be at most 500 USD. Available service offers will be described in a similar fashion. During service discovery, the DSD matcher will compare available offers with the request. For this comparison the request tree is traversed and for each set the matcher determines whether the set in the offer is a subset of the set in the request, thereby ensuring that the effect provided by the service is one that the requestor really wanted. This comparison can be done quite efficiently and results in very precise matching results. This is one of the strengths of DSD compared with other matchmaking algorithms: Typically, they either take not all the information avaiable in the offer into account or they are not efficient (most of the time not even decidable).

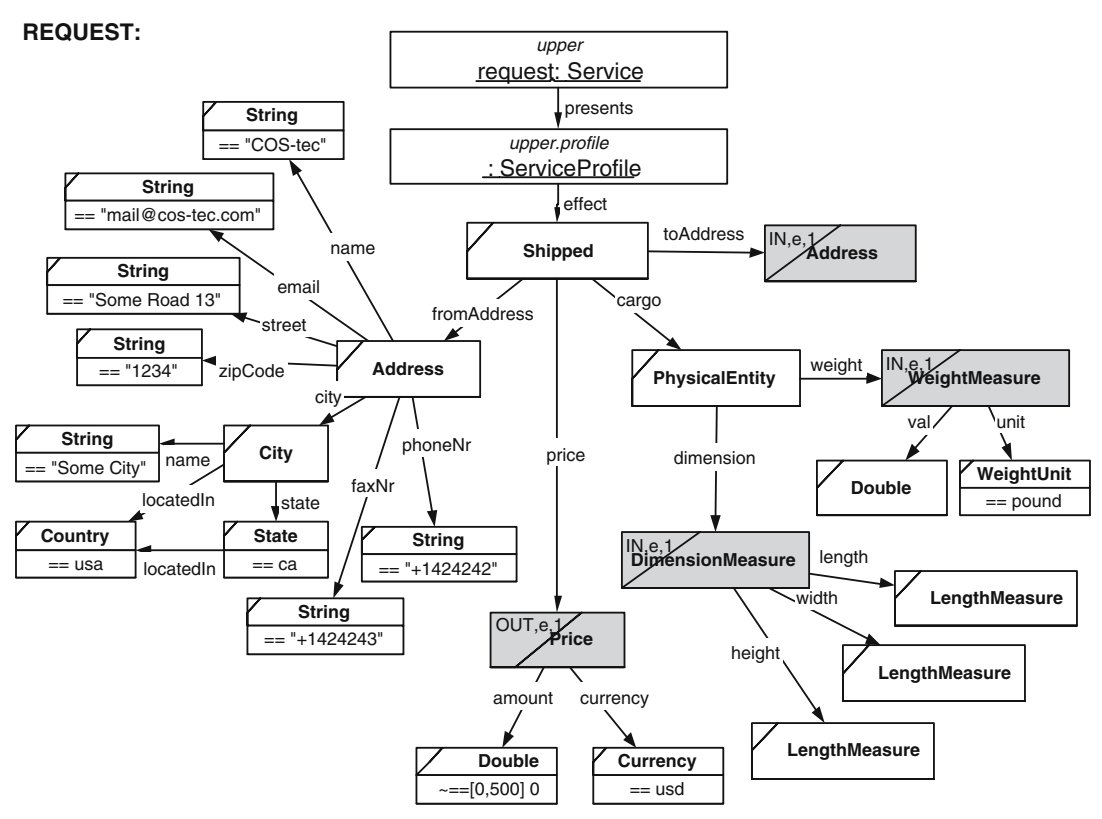

Fig. 2. Example request for a shipment service (some details have been omitted)

\section{Integrating DSD into BPEL Processes}

The basic idea how to integrate the DSD-Middleware into a BPEL process is to make it available through a web service interface. The BPEL process can then send service requests to that interface, thereby invoking the DSDMiddleware which will locate available offers, find the best suitable one and call the corresponding service provider with appropriately configured parameters. This architecture is shown in Figure 3. The major difficulty involved in this approach is to provide easy to handle yet powerful and expressive mapping mechanisms to translate the XML-based legacy data representation of the BPEL process into ontology based semantic request descriptions understood by 


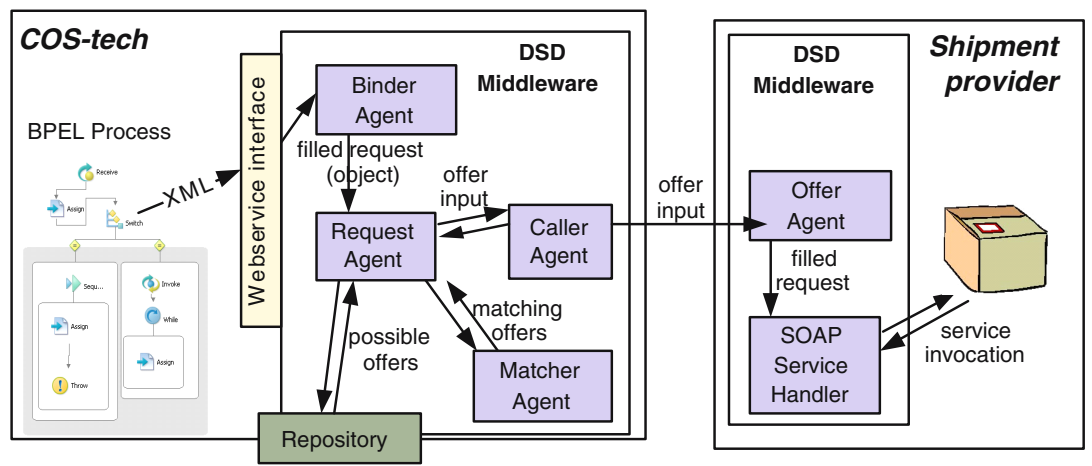

Fig. 3. Overview of implemented architecture

the DSD-Middleware. In the following we will first explain this mechanism and then give some more detail about how the DSD-Middleware works.

\subsection{Mapping from Legacy Data to Semantic Request Descriptions}

We anticipate that for most use cases the structure of regularly occurring requests like our shipment service discovery requests (compare to Figure 2) will remain fairly stable, but parameters and variable values will change. We therefore decided not to build a request from scratch within the BPEL process but to rather deploy a request template to the DSD-Middleware at design time which will be filled with variable values at runtime. This way the creation of the request description can be done in the editors designed for that within the DSD framework and is completely separated from the existing BPEL legacy order management. All the order management has to do and all that has to be changed in the BPEL process is that the process needs to send the name of the request template to use and the parameters (like address, package weight, package pickup time...) in BPEL friendly XML format to the webservice interface of the middleware. The format of those parameters (i.e. the XML schema) may be defined by the legacy BPEL process and the middleware therefore adapts to the existing process and not vice versa.

The BinderAgent component of the middleware is responsible for the management of deployed request templates. It will be called by the webservice interface, locate the template corresponding to a given request name and transform the given input XML parameters to ontological concepts and instances represented as Java objects (the data format used internally by the middleware). The information how to do that transformation can be defined declaratively in the request template's grounding by specifying XmlDsdMapping instances as shown in Figure 4. Each mapping identifies a variable from the request and a node from the given XML parameters that is used to fill that variable. The node is specified using an arbitrary XPath expression; this offers great flexibility. If the 
expression evaluates to a node list instead of a single node, multiple instances of the variable to fill will be created as necessary.

Where necessary a custom converter class and method name may be optionally specified which will be used by the middleware to unmarshall the contents of the node into DSD variables. The price variable for instance is filled with data taken from the "maximumPrice" child element of the given parameters. The unmarshalling is provided by invoking the "convertToPriceDescription" method of the "MaxPriceConverter" class.

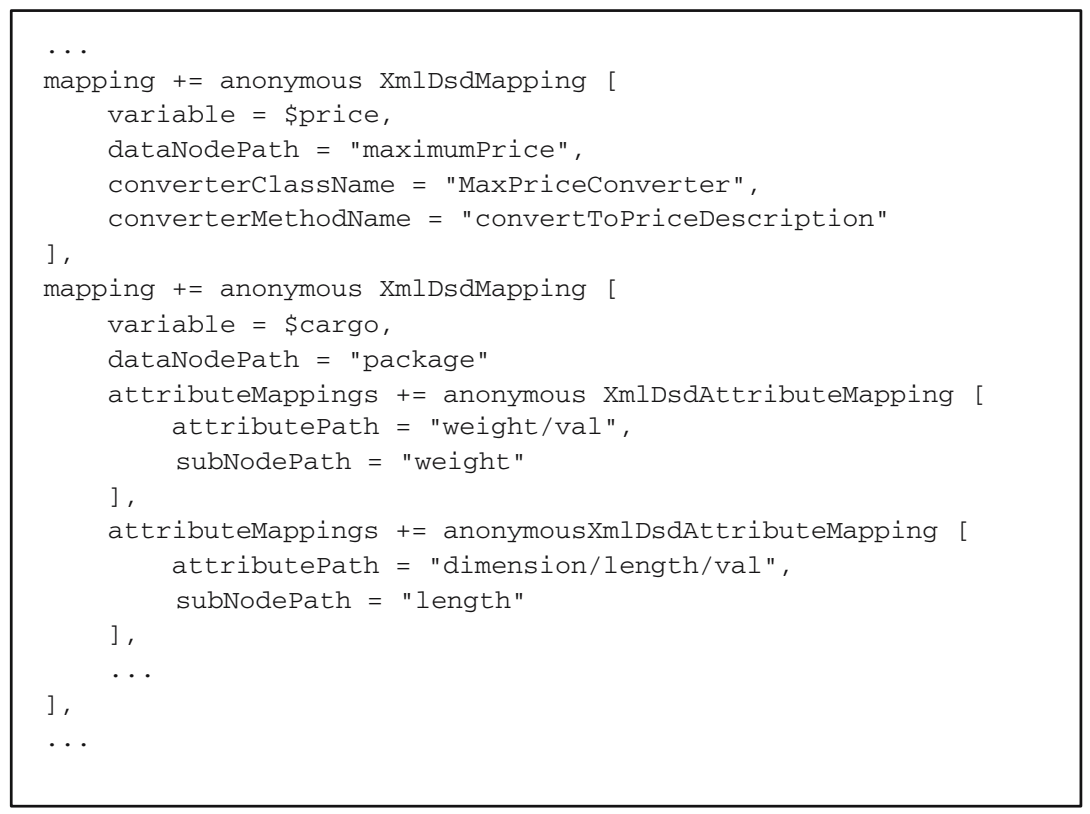

Fig. 4. Excerpt from mapping definitions XML to DSD variables

Usually the deployment of such custom converter classes is not necessary. If no custom converter class is given, two cases need to be distinguished: If the variable to fill is of a simple type (corresponding to XML Schema's atomic types), standard marshalling will be used to fill it using the value from the specified node. If the variable has a complex type, an empty variable of that type will be initialized and XmlDsdAttributeMapping instances have to be provided that define how its attributes will be filled. These submappings work similar to the XmlDsdMappings but operate in the context of the variable and node specified by the parent mapping. As XmlDsdAttributeMappings can be nested arbitrarily deep this mechanism is flexible enough to deal with any content, including nested lists. As shown in Figure 4 the "cargo" variable will be filled with data from the "package" child element of the given parameters. If multiple "package" child elements are found, multiple "cargo" variables will be created. The fillings of 
the "cargo" variable's attributes are defined by the given attributeMappings. The "weight" attribute's "val" attribute for instance (compare to Figure 2) will be filled using the data from the child element "weight" of the node that had been identified by the "package" XPath expression. Since the type of the "val" attribute is a simple double type and no converter class is specified, standard unmarshalling will be used. As shown in the next mapping definition, variable attributes may be identified using concatenated paths. In this case the "val" attribute of the "length" attribute of the "dimension" attribute of the "cargo" variable is filled (Note that this attribute has been omitted in Figure 2 for the sake of clarity).

\subsection{Request Execution by the DSD-Middleware}

Once the BinderAgent has translated the input XML to DSD variables and filled the request template accordingly, that request is forwarded to the RequestAgent. The RequestAgent contacts the Repository 1 in order to obtain a list of available offer descriptions. Together with this list the request is then forwarded to the MatcherAgent. The MatcherAgent forms the heart of the middleware. Not only does it provide efficient semantic matching of the request against the offers, it also configures the offers optimal with regard to the request. More details about the matching process can be found in 3 . An important property of the MatcherAgent is its ability to interact with a service provider during matchmaking. In the description of its offer a shipment service provider can, e.g., specify that the price of a shipment may be inquired by calling a getPrice operation with the size of the package to send as parameter. The MatcherAgent is then able to automatically call that operation and use the dynamically provided additional information in the matching process.

Upon completion of the matching process the MatcherAgent returns a list of properly configured service offers to the RequestAgent, ordered by how well the offers suit the request. In the most basic case of our shipping scenario the least expensive service would rank highest, in more advanced scenarios preferences concerning pickup times, speed of delivery and other properties can be included.

The RequestAgent picks the best offer and forwards it to the CallerAgent which invokes the OfferAgent at the middleware instance running on the service provider's server. Based on information provided in the offer description's grounding the OfferAgent has to bridge the gap between the semantic description of the offer and its actual implementation. Different implementations (like Java program, web service, ...) are mirrored by corresponding different types of groundings in the offer's service description. Depending on the type of the grounding found in that description, the OfferAgent thus delegates the invocation to the appropriate ServiceHandler. In order to perform the service invocation the ServiceHandler has to translate the given configured service offer description into a service invocation. In case of a web service the input parameters of that service have to be extracted from the configured offer description and transformed

\footnotetext{
${ }^{1}$ The implementation of the repository is pluggable and independent from the rest of the middleware. It is therefore not within the scope of this paper.
} 
into an XML message the service can process. This is the complementary task of the translation performed by the BinderAgent. Besides providing essential information like the address of the service, the offer grounding therefore also has to define mappings which are this time used the other way round, namely to create XML data from DSD variables analogously to the proceeding explained in Section 3.1. In a similar fashion the reply of the web service is transformed back into DSD variables. These are transferred back through the middleware and returned to the calling BinderAgent. Finally, the BinderAgent transforms them into an XML message that is replied to COS-tec's BPEL order management process. Once again these transformations are performed using mapping definitions from the offer and request's grounding.

The system introduced in this paper has been implemented and tested. An elaborate scenario including selection, binding and invocation of a shipping service has been implemented in the context of the 2006 Semantic Web Services Challenge 4].

\section{Evaluation}

We offer an easy and lightweight way to enable dynamic binding for the component services used by BPEL processes. Our approach is beneficial, if several different providers competitively offer a certain necessary functionality used by the BPEL process (like shipment ordering, stock quote lookup, component purchasing, ...). In this case our approach enables the BPEL process to always use (i.e. choose and invoke) the best provider on a case by case basis. Thereby the different services do not have to adhere to a standardized protocol or data representation to enable to be bound and invoked automatically, but of course need to annotate or describe their service semantically using an agreed upon language - in this case DSD. To keep the approach lightweight, only limited support is currently offered for cases where one component service would have to be replaced by a whole workflow of other services. A discussion of this topic of automatic composition synthesis is beyond the scope of this paper.

BPEL uses the notion of partner links, roles and partners to identify conversations with varying actual services. It may be necessary to ensure that the same single partner service, once chosen, will be used in later calls to the same role. This is somewhat contrary to the idea of dynamic binding but if necessary can easily be ensured by giving the provider name as parameter in later calls of the DSD-Middleware. This will effectively limit the possible matches to the single provider registered with the given name. More flexible limitations of possible matches for dynamic bindings downstream the business process execution can be achieved similarly. This way one could ensure that certain providers are always (or never) used in combination.

During runtime the integrating of the DSD requests into BPEL processes includes up to four data transformations: The input from the BPEL process has to be transformed to DSD in order to be processed by the middleware. It has then to be transformed to a format understood by the chosen provider's 
implementation to invoke the service. Finally, output data has to be transformed the same way back. Although this might look like too much overhead, it is unavoidable to overcome heterogeneities regarding data representation and choreography between COS-tec's legacy process on the one hand and the various systems of many independent shipment service providers on the other hand. An $\mathrm{xml}$ message issued by COS-tec cannot be used directly to communicate with a dynamically discovered service provider since this would require a unified $\mathrm{xml}$ interface for all the various potential providers.

By supporting the necessary translations through declarative specifications within the semantic description of a service request or offer, the bulk of actual work concerning these translations can be performed by the DSD middleware and doesn't have to be implemented by the participants (COS-tec or the shipment service providers). Furthermore, by putting the translation rules into the description's groundings they are cleanly separated from other concerns and can be easily adapted, if a participant were to change its data format. If, for instance, one of the shipment service providers changes its data format, all it has to do is to publish an updated version of its offer description to the repository system used. COS-tec's order management system could still use that provider without any change, in fact COS-tec wouldn't even notice a change took place!

The approach presented in this paper could in principle be realized using any service description language that allows for a precise encoding of user preferences in the request and provides a matching algorithm that is able not only to find the most appropriate offer, but also to directly invoke it. While a detailed comparison of DSD with other such languages and discovery techniques is outside the scope of this paper, it can be found in 3 . There, we reach the conclusion that neither WSMO nor OWL-S, the two most widespread such languages currently meet these requirements. Extensions of these languages, however, would be a suitable basis for this approach. In the following section we will give a short overview of the related work that also tries to support dynamic binding for business processes.

\section{Related Work}

The work closest to the one presented in this paper is by Mandell and McIllraith 5. They also propose to integrate dynamic service discovery and binding into BPEL processes. Discovery and matchmaking is performed by querying a knowledge base of DAML-S service profiles with requests expressed in the DAML Query Language (DQL). This machinery is then made availabe to BPEL processes through a "Semantic Discovery Service" (SDS). Although this idea is very similar to ours, the realization is not. The SDS is agnostic to the content of the service descriptions and invocation messages it receives. An invocation message of the SDS consists of two parts: The abstract service request description to be sent to the DAML-S knowledge base and the parameters to be sent to a discovered service. Both are simply forwarded. Thus the calling BPEL process has not only to deal with semantic descriptions directly, it also has to output parameters in a format that will be understood by the discovered services. This 
is very different from the configurable, adaptable mappings approach presented in this paper.

The METEOR-S project [6] aims at creating a framework to support dynamic selection of optimal partner web services. Developers create abstract processes that contain service templates (semantic requests). At runtime a configuration module binds these to concrete services using semantic discovery and an execution environment handles their invocation. Unlike the previously mentioned approach METEOR-S also deals with the problem of data mapping. Similar to our approach, developers may specify mappings between each element of an input data of a web service and the corresponding ontological concept. Compared to our work the way how the semantic technology is integrated by METEOR-S differs. While we provide a web service gateway to the DSD Middleware that enables to replace single (or multiple) statically bound service invocations by calls of the middleware, the METEOR-S framework takes whole abstract processes as input to transform it into a concrete process observing global constraints and a global optimization function. Thus, the targeted use case is somewhat different. But more importantly, the SWRL reasoner used by METEOR-S to perform this task is not as powerful as the DSD MatcherAgent as it lacks the ability to express user preferences and to invoke service operations during matchmaking to gather dynamic information (This difference applies to [5], too).

Just recently Lemcke and Drumm [7] introduced a work to show the benefits of integrating semantic Web service technologies into business processes. Just like this paper they use a logistics scenario and aim to support dynamic carrier selection. However, they use a set of predefined carriers to create a single business process for each of them at design time. At runtime they then use semantic technology to pick and instantiate the most appropriate process. Although the use case is the same, the focus of this work is less on discovery and binding and more on automated mapping of in- and output parameters to support the creation of the business processes at design time.

The work by Oberle et al. 8, makes a case for the necessity to trade of between the high maintenance costs of the WS-* approaches and the high modeling cost of semantic approaches. It thus suggests a compromise that explicitly does not aim at automating anything but only the most efficient things. We share this idea and the underlying motivation, but the resulting work aims at very different objectives. Based on a set of use cases Oberle et al. try to identify who could benefit to what extent from what kind of semantic modeling of web services. Thereby they aim at examining how to support the developers of web applications by exploiting which kind of semantic information. Thus, they focus on the usage of semantic technology during process design time, while we leverage semantic technology at process runtime.

\section{Summary and Conclusion}

In this paper we have shown how existing service-oriented applications can leverage the advantages offered by novel semantic web service technology without 
compromising trust in the application or encountering unreasonable effort during the transition.

The basic idea is to enable BPEL processes to bind to the semantics-based DSD middleware instead of statically binding to an external service provider. In places where the static binding is replaced by the DSD middleware binding, predefined templates will be used to issue semantic requests. The DSD middleware then uses its semantic technology to dynamically find and invoke the most suitable service provider from a potentially vast pool of candidates. This dynamic binding results in a robust system that always uses the best provider of functionality available without the need of manual intervention. Developers can choose at which steps of a BPEL process to replace the original static binding by the DSD-based one. This leaves the control about how much semantics to introduce in the discretion of the developer. This overcomes both the widespread lack of trust in semantic technology and the high entrance costs associated with a complete migration to semantic technology.

The approach presented in this paper offers an opportunity for a smooth transition from legacy web service applications to more powerful semantic web service technology. We believe such a seamless transition to be a necessary prerequisite for the adaptation of semantic service technology in real-life commercial settings.

\section{References}

1. Sprott, D., Wilkes, L., Veryard, R., Stephenson, J.: Web services roadmap. In: CBDI. Report Series - Guiding the Transition to Web Services and SOA (2003)

2. W3C World Wide Web Consortium: (www.w3c.org)

3. Klein, M., König-Ries, B., Müssig, M.: What is needed for semantic service descriptions - a proposal for suitable language constructs. International Journal on Web and Grid Services (IJWGS) 1(3/4), 328-364 (2005)

4. Küster, U., König-Ries, B., Klein, M.: Discovery and mediation using diane service descriptions. In: Second Workshop of the Semantic Web Service Challenge 2006, Budva, Montenegro, June 2006 (2006)

5. Mandell, D.J., McIlraith, S.A.: Adapting BPEL4WS for the semantic web: The bottom-up approach to web service interoperation. In: Fensel, D., Sycara, K.P., Mylopoulos, J. (eds.) ISWC 2003. LNCS, vol. 2870, Springer, Heidelberg (2003)

6. Verma, K., Gomadam, K., Sheth, A.P., Miller, J.A., Wu, Z.: The METEOR-S approach for configuring and executing dynamic web processes. Technical Report 624-05, LSDIS Lab, University of Georgia, Athens, Georgia, USA (2005)

7. Lemcke, J., Drumm, C.: Semantic business automation. In: Proceedings of the 3rd European Semantic Web Conference's Industry Forum, Budva, Montenegro (2006)

8. Oberle, D., Lamparter, S., Eberhart, A., Staab, S.: Semantic management of web services. In: ICWS05. Proceedings of the 2005 IEEE International Conference on Web Services, Orlando, Florida, IEEE Computer Society Press, Los Alamitos (2005) 